1 A Retrospective Validation Study of 3 Models to Estimate the Probability of

Oncology Follow-up Centre

4

\title{
5 Introduction
}

7 The management of patients with small pulmonary nodules (PNs) is clinically

8 challenging. With the widespread availability of multi-detector row CT scanners

9 and the significant increase in the number of scans performed ${ }^{1}$, this scenario is

10 likely to increase in clinical practice, and clear evidence is required to guide 11 management strategies.

13 Data from published case series in Europe ${ }^{2} 3$, Asia ${ }^{4}$, and America 78 report the 14 prevalence of PNs from $2 \%$ to $24 \%$ (mean 13\%) with a mean lung cancer 15 prevalence of $1.5 \%$. The prevalence of PNs in a screening population is higher 16 (mean 33\% ${ }^{9}$ but management of nodules in this scenario is predetermined for 17 the majority of screening programmes.

19 The effective management of PNs requires knowledge of the "pre-test" 20 probability of malignancy in each individual patient, prior to conducting further 21 investigation ${ }^{10}$. The British Thoracic Society Guidelines for the management of 22 pulmonary nodules recommends the use of prediction models when evaluating 23 these patients. 
25 Twenty-eight studies $11-33$ in the literature have evaluated the clinical and

26 radiological characteristics of $\mathrm{PNs}$ in relation to the probability of malignancy.

27 Four studies have developed composite prediction models that can be used in 28 clinical practice and are easily accessible.

30 Swensen et al ${ }^{11}$ (Mayo model) and Gould et al 12 (VA model) identified

31 independent clinical and radiological predictors of malignancy and used this to 32 create and validate models with an AUC of 0.8.

34 The Mayo model was validated with the additional parameter of PET avidity by 35 Herder et al ${ }^{13}$, increasing the AUC to 0.92 (the Herder model).

37 McWilliams et al $^{14}$ developed a model based on a lung cancer screening 38 population. The AUC for this model was 0.94 and specifically 0.91 for nodules $39 \leq 10 \mathrm{~mm}$.

41 A number of case series have attempted to address the likelihood of malignancy 42 in patients with known extra-thoracic cancer 34-35. However due to their 43 heterogeneity, and small patient numbers these studies do not provide sufficient 44 data to distinguish benign from malignant nodules or a metastasis from a 45 primary lung cancer.

47 The aim of this study was to validate three existing composite prediction models 48 in a non-screening clinically referred patient population with small, 49 predominantly sub-centimeter PNs. 


\section{$50 \quad$ Materials and Methods}

51

52 The Research and Development department at our Trust approved the study and

53 waived requirements for informed consent.

54

55

Case Identification and Data Collection

56

57 The electronic medical records of all patients with PNs reported on CT imaging,

58 between January 2012 to January 2013 at our centre were identified by a word

59 search on the Radiology Information System (RIS) using the phrase "pulmonary

60 nodule(s)" and/or "lung nodule(s)". Once identified, each report was reviewed

61 and patients with nodules of less than $12 \mathrm{~mm}$ identified. A $12 \mathrm{~mm}$ nodule

62 diameter was used as a cut-off to maximize the number of primary lung cancers

63 (which often present above $10 \mathrm{~mm}$ diameter at initial presentation) included

64 with the data-set.

65

66 Patient demographics, the clinical indication for CT imaging, radiological

67 characteristics of the pulmonary nodules and the final diagnosis were recorded.

68

69 The patients were divided into two groups:

70

71 1. Incidental finding on a chest CT in patients with or without respiratory symptoms with any history of malignancy within 5 years. 
94 The initial electronic search of the radiology information search system

95 identified 2256 CT scans performed in 1209 patients. 96

2. Patients with either a known or prior cancer within the last 5 years, scanned either as a staging or follow up scan, or scanned for another reason.

The distribution of patients in each group is shown in Table 1.

Ground truth diagnosis of the pulmonary nodule was determined as:

a) Malignant when histopathology revealed a specific malignant diagnosis.

b) Pulmonary metastasis without histology was defined as a non-calcified nodule with a smooth regular border, demonstrating either growth (percentage volume change of $\geq 25 \%{ }^{34}$ ), or a reduction in size due to chemotherapy treatment, assessed by a chest radiologist.

c) Benign diagnosis by either benign histopathology or nodule stability over 2 years of CT follow-up, defined as a percentage volume change of $\leq 25 \%$. (1)

\section{Patients} 3 
97 The CT image was reviewed to confirm the presence of the nodule, confirm

98 nodule size (using 2D caliper measurements) and to collect information about

99 nodule morphology. In the case of nodules not diagnosed as malignant on 100 histology, volume growth was recorded using the Lung VCAR application (GE 101 Healthcare systems).

103 The electronic patient records were reviewed for patient age, gender, smoking 104 history, history of active or previous cancer, and family history of cancer. 105 Histology reports were reviewed to confirm the diagnosis of the resected nodule.

107 Patients were excluded for the following reasons: incomplete demographic data, 108 no nodule identifiable on the CT, nodule size $>12 \mathrm{~mm}$, CT follow up of $<2$ years 109 in the case of likely benign nodules or if no histology was available.

111 Figure 1 summarises study eligibility and reasons for patient exclusion.

\section{Data Analysis}

115 A comparison of final diagnosis to the probability of malignancy predicted by 116 each of the Mayo Model, the VA model and the McWilliams model was calculated 117 (see Appendix A for links to each prediction model calculator). Model accuracy 118 was assessed by calculating the area under the receiver operating characteristic 119 (ROC) curve, comparing the model probability of malignancy with the actual 120 patient diagnosis. Calibration curves were calculated between the observed and 121 predicted probabilities of the models (see Appendix B). 
123 Attempted assessment of the Herder model was limited by the small number of

124 patients undergoing PET-CT for sub-centimeter PNs (required in the model). The 125 results of this analysis are presented in Appendix B only.

126

127 Analyses were performed using SPSS for Mac Version 22.0.

128

129

130

131

132

133

134

135

136

137

138

139

140

141

142

143 
144 Results

146702 of 1209 patients met the inclusion criteria for the study.

148 Baseline demographics of the population are presented in Table 1. Current or 149 former smokers accounted for 457 (65\%) patients and 306 (43.6\%) patients had 150 an active or previous history of lung or extra-thoracic cancer.

151

152 Of 702 nodules identified, 379 (54\%) were benign, 303 (43\%) pulmonary 153 metastases and $20(3 \%)$ primary lung cancers. The overall prevalence of 154 malignancy in this cohort was $46 \%$.

155

156 Size of the nodule was not associated with a malignant diagnosis $(\mathrm{p}=0.076)$. The 157 majority of nodules were solid (95.7\%) and all perifissural nodules were benign. 158 Nodules in upper lobes were more likely to be malignant in comparison to 159 nodules found in the lower lobes $(\mathrm{p}=0.021)$, but there was no significant 160 difference in the number of malignant nodules seen in the right lung.

1617 of the 379 patients (\%) with a benign PN had a diagnosis of fungal infection 162 confirmed by histopathology.

164 Of the 20 primary lung cancers, 16 were adenocarcinomas and 4 squamous cell 165 carcinomas. Patients with a prior history of malignancy were more likely to 166 present with a malignant nodule $(\mathrm{p}=0.001)$. The diagnosis of the primary cancer 167 in patients with pulmonary metastases in shown in Figure 2. 
168

169 A summary of the other characteristics and method of nodule diagnosis is shown

170 in Table 2.

171

172

173

174

175

176

177

178

179

180

181

182

183

184

185

186

187

188

189

190

191 
194 The probability of malignancy score was calculated by each model using online 195 published calculators (Appendix A). Probability scores were compared to the 196 actual patient diagnosis determined by histopathology or 2 year CT thorax 197 follow-up of the pulmonary nodule.

\section{Comparison Between Models}

200

201 The area under the ROC curve (AUC) was highest for the McWilliams Model $202(0.82 ; 95 \%$ CI 0.78-0.91) and lowest for the Mayo Model (0.58; 95\% CI 0.55203 0.59). The VA Model had an AUC of (0.62; 95\%CI 0.47-0.64).

204 The ROC curves for each of the models are shown in Figure 3.

205

206 The AUC for each of the models was calculated separately for patients with a 207 prior history of malignancy (within 5 years) and for those without a prior history 208 of malignancy. This analysis showed that the McWilliams model performed less 209 well in patients with a prior history of malignancy, whilst the Mayo and VA 210 performed slightly better in this group.

211 The results are shown in Table 3.

213 The calibration of the models is shown in Figure 4, Appendix B. 


\section{Discussion}

218 This is the largest study to our knowledge to validate predictive models for PNs

219 in a non-screening clinically referred patient population. All three of the 220 validated models in this study were not specifically designed for use in patients 221 with a previous history of malignancy within 5 years of presentation with a PN. 222 However in clinical practice, it is often this group of patients where it is 223 challenging to differentiate between primary lung cancer, pulmonary metastases 224 or benign nodules, and this is particularly the case with sub-centimeter nodules. 225 In this study population, $12.1 \%$ of patients with a prior history of malignancy 226 were subsequently diagnosed with a benign nodule.

228 Our findings indicate that the accuracy of all models is lower in patients with 229 prior malignancy than that reported in the papers that described their 230 development. The McWilliams model was the most accurate overall; this may be 231 due to this model having a high percentage of sub-centimeter pulmonary 232 nodules in both development and validation datasets.

234 The accuracy of the Mayo and VA models was higher in patients with a prior 235 history of malignancy. This may be due to the higher prevalence of nodule 236 malignancy in the models' development datasets, similar to our population. 237 Conversely, the McWilliams model (based on a lung cancer screening 238 population) had a greater accuracy amongst patients without a previous history 239 of cancer. 
241 All models underestimated the probability of malignancy from the first up to at

242 least the third quintile. This may be due to the prevalence of nodule malignancy

243 in our patient sample being higher (46\%) than the development data set of the

244 Mayo (23\%) and McWilliams (5.5\%) model. In contrast, the VA model was

245 developed from patient cohorts with a relatively high prevalence of malignancy

246 (54\%). Hence the highest overestimation for malignancy was seen in this model,

247 in those with a high pre-test probability.

249 The calibration plots (Appendix B, Figure 4) demonstrate variability in the 250 predictive ability of each model according to malignant nodule prevalence. This 251 implies that before widespread use of predictive models as suggested by the 252 current evidence based guidelines, the characteristics of the local population 253 should be assessed. The overall appearance of the McWilliams model calibration 254 plot showed a greater spread between each quintile in comparison to the other 255 models, demonstrating it to be the most accurate at predicting malignancy for 256 nodules with both a high, intermediate and low probability.

258 Combining information from the ROC analysis and calibration data, the 259 McWilliams model has the best discriminative ability between benign and 260 malignant nodules using these validation methods. It performs best overall in 261 patients without a prior history of malignancy (Table 3).

263 The first study to validate prediction models in the UK by Al-Ameri et $\mathrm{al}^{36}$ found 264 the Herder to be the most accurate in predicting malignant risk, even in patients 
with a prior history of cancer. For smaller sub-centimeter nodules, the highest accuracy was seen for the McWilliams model, as seen in this study.

268 The BTS pulmonary nodule guidelines 2015 recommend the validation of 269 composite prediction models in patients with a prior history of extra-thoracic 270 malignancy. Our results demonstrate the limitations of these composite models 271 when utilised in a population with a high prevalence of such patients and in 272 particular in PNs $\leq 12 \mathrm{~mm}$. Further research is needed to risk stratify patients 273 according to the patient's mode of presentation, irrespective of nodule size. 274 Obtaining data from multiple centres may reduce bias in the distribution and 275 prior diagnoses of patients.

277 This study has several limitations. There was an under-representation of 278 primary lung cancers within the population of patients presenting with small 279 (particularly sub-centimeter) pulmonary nodules. This is representative of a non-screening clinical practice, with most patients with clinically diagnosed 281 primary lung cancer presenting at a later stage. The population had a high 282 proportion of patients with pulmonary metastases, as patients were included 283 with a current or prior history of cancer which is a common and difficult clinical 284 scenario. There was therefore a bias towards these diagnoses which may be 285 underrepresented in other centres. The majority of patients with pulmonary 286 metastases did not have a histological diagnosis. In addition, there were no 287 patients from a lung cancer screening population, as this is yet to be established 288 in the UK. However, the selected population reflects clinical practice in whom 289 there is likely to be a mix of patients with and without a prior history of cancer, 
290 suggesting that our results are likely to be applicable to a broad range of UK 291 practice.

292

293 In conclusion, our study demonstrates that all three prediction models assessed 294 had a lower predictive value than published in the original papers. The 295 McWilliams model appears to be the most clinically useful tool to establish the 296 pre-test probability of malignancy in an unselected population that includes 297 patients with a prior history of malignancy.

298 Further studies are now needed to create and validate prediction models 299 tailored specifically for patients with a prior history of extra-thoracic 300 malignancy.

301 
Figure Legends:

316

317 Figure 1: Study Eligibility and Reasons for Patient Exclusion

318

319 Figure 2: Primary Cancer Site of Patients with Pulmonary Metastases

320

321 Figure 3: Receiver Operator Characteristic Curves for Validation of 4 Composite

322 Prediction Models

323

324 Figure 4: Calibration Curves for Validation of Composite Prediction Models (in

325 Appendix B)

326

327

328

329

330

331

332

333

334

335

336

337

338 
1 Costello P, Anderson W, Blume D. Pulmonary nodule: evaluation with spiral volumetric CT. Radiology 1991;179:875876 ,

2 Cardinale L, Cortese G, Borasio P et al: Low dose CT in early lung cancer diagnosis: Prevalence data. Radiologica Medica $2005 ; 110: 532-43$

3 Clin B, Luc A, Morlais F et al: Pulmonary nodules detected by thoracic computed tomography scan after exposure to asbestos: Diagnostic significance. International Journal of Tuberculosis and Lung Disease 2011; 15 (12): 1707-1713

4 Hanamiya M, Aoki T, Yamashita Y et al: Frequency and significance of pulmonary nodules on thin-section CT in patients with extrapulmonary malignant neoplasms. European Journal of Radiology 2012; 81:152-7

5 Kim YH, Lee KS, Primack SL et al: Small pulmonary nodules on CT accompanying surgically resectable lung cancer: likelihood of malignancy. Journal of Thoracic Imaging 2002 ;17:40-6

6 Iribarren C, Hlatky MA, Chandra M et al: Incidental Pulmonary Nodules on Cardiac Computed Tomography: Prognosis and Use. American Journal of Medicine 2008; 121 (11):989-996

7 Khokhar S, Vickers A, Moore MS et al: Significance of non-calcified pulmonary nodules in patients with extrapulmonary cancers. Thorax 2006;61:331-6

8 Margolis ML, Howlett P, Bubanj R: Pulmonary nodules in patients with esophageal carcinoma. Journal of Clinical Gastroenterology 1998; 26:245-8

9 British Thoracic Society Pulmonary Nodule Guidelines. Thorax doi 10.1136/thoraxjnl-2015-207221

10 Greenberg AK, Lu F, Goldberg JD et al: CT scan screening for lung cancer: Risk factors for nodules and malignancy in a high-risk urban cohort. PLoS ONE 2012; 7 (7)

11 Swensen SJ, Siverstein MD, Ilstrup DM et al: The probability of malignancy in solitary pulmonary nodules. Application to small radiologically indeterminate nodules. Archives of Internal Medicine 1997;157:849-55

12 Gould MK, Ananth L, Barnett PG et al: A clinical model to estimate the pretest probability of lung cancer in patients with solitary pulmonary nodules. Chest 2007;131:383-8

13 Herder GJ, van Tinteren H, Golding RP et al: Clinical prediction model to characterize pulmonary nodules: validation and added value of 18F-fluorodeoxyglucose positron emission tomography. Chest 2005;128:2490-6

14 McWilliams A, Tammemagi MC, Mayo JR et al: Probability of cancer in pulmonary nodules detected on first screening CT. N Engl J Med 2013; 369:910-9

15 Mery CM, Pappas AN, Bueno R et al: Relationship between a history of antecedent cancer and the probability of malignancy for a solitary pulmonary nodule. Chest 2004;125:2175-81

16 Quint LE, Park CH, Iannettoni MD et al: Solitary pulmonary nodules in patients with extrapulmonary neoplasms. Radiology 2000; 217:257-61 
17 Smyth EC, Hsu M, Panageas KS et al: Histology and outcomes of newly detected lung lesions in melanoma patients. Annals of Oncology 2012;23:577-82,

18 de Hoop B et al: Pulmonary perifissural nodules on CT scans: rapid growth is not a predictor of malignancy. Radiology 265:611-6, 2012

19 Ahn MI et al: Perifissural nodules seen at CT screening for lung cancer. Radiology 254:949-56, 2010

20 Franquet $\mathrm{T}$ et al: Infectious pulmonary nodules in immunocompromised patients: usefulness of computed tomography in predicting their etiology. Journal of Computer Assisted Tomography 27:461-8, 2003

21 Gurney JW: Determining the likelihood of malignancy in solitary pulmonary nodules with Bayesian analysis. Part I. Theory. Radiology 186:405-13, 1993

22 Gurney JW et al: Determining the likelihood of malignancy in solitary pulmonary nodules with Bayesian analysis. Part II. Application. Radiology 186:415-22, 1993

23 Dewan NA et al: Likelihood of malignancy in a solitary pulmonary nodule: comparison of Bayesian analysis and results of FDG-PET scan. Chest 112:416-22, 1997

24 Edinburgh KJ et al: Multiple pulmonary nodules in AIDS: usefulness of CT in distinguishing among potential causes. Radiology 214:427-32, 2000

25 Harders SW et al: High resolution spiral CT for determining the malignant potential of solitary pulmonary nodules: refining and testing the test. Acta Radiologica 52:401-9, 2011

$26 \mathrm{Kim} \mathrm{H}$ et al: Predictors for benign solitary pulmonary nodule in tuberculosis-endemic area. Korean Journal of Internal Medicine 16:236-41, 2001

27 Kui M et al: Evaluation of the air bronchogram sign on CT in solitary pulmonary lesions. Journal of Computer Assisted Tomography 20:983-6, 1996

$28 \mathrm{Li} \mathrm{F}$ et al: Malignant versus benign nodules at CT screening for lung cancer: comparison of thin-section CT findings. Radiology 233:793-8, 2004

$27 \mathrm{Li} \mathrm{Y}$ et al: Development and validation of a clinical prediction model to estimate the probability of malignancy in solitary pulmonary nodules in Chinese people. Clinical Lung Cancer 12:313-9, 2011

28 Malaisamy S et al: The clinical and radiologic features of nodular pulmonary sarcoidosis. Lung 187:9-15, 2009

29 Saito $\mathrm{H}$ et al: Usefulness of circumference difference for estimating the likelihood of malignancy in small solitary pulmonary nodules on CT. Lung Cancer 58:348-54, 2007

30 Schultz EM, Sanders GD, Trotter PR, et al: Validation of two models to estimate the probability of malignancy in patients with solitary pulmonary nodules. Thorax 63:335-41, 2008

31 Swensen SJ et al: Solitary pulmonary nodules: clinical prediction model versus physicians. Mayo Clinic Proceedings 74:319-29, 1999 
32 Yonemori $\mathrm{K}$ et al: Development and validation of diagnostic prediction model for solitary pulmonary nodules. Respirology 12:856-62, 2007

33 Copp DH et al: Clinical and radiologic factors associated with pulmonary nodule etiology in organ transplant recipients. American Journal of Transplantation 6 (11):2759-2764, 2006

34 Khokhar et al 2006. Significance of non-calcified pulmonary nodules in patients with extrapulmonary cancers. Thorax $2006 ; 61: 331-336$

35 Hanamiya et al. Frequency and significance of pulmonary nodules on thin-section CT in patients with extrapulmonary malignant neoplasms. European Journal of Radiology 2012 ; 81:152-157

36 Ameri et al: Risk of malignancy in pulmonary nodules: A validation study of four prediction models. Lung Cancer $89(1): 27-30,2015$ 